\title{
Improvement Performance of Planar Perovskite Solar Cells by Bulk and Surface Defect Passivation
}

Qingbin Cai, Zhichao Lin, Wenqi Zhang, Guibin Shen, Xiaoning Wen, Hongye Dong, Xiangning $\mathrm{Xu}$, Dongping $\mathrm{Zhu}$, and Cheng $\mathrm{Mu}^{*}$

Department of Chemistry, Renmin University of China, Beijing 100872, P. R. China.

*E-mail: $\underline{\text { cmu@,ruc.edu.cn }}$

\section{Table of Contents: (5 tables and 6 figures)}

Table S1. The fitted parameters of TRPL spectra (excitation at $475 \mathrm{~nm}$ ) of Control, $\mathrm{CsCl}$ and $\mathrm{CsCl} / \mathrm{CH}_{3} \mathrm{O}$-PEAI perovskite films deposited on quartz glass.

Table S2. Calculated perameters and trap density (Nt) of the Control, $\mathrm{CsCl}$ and $\mathrm{CsCl} / \mathrm{CH}_{3} \mathrm{O}-$ PEAI perovskite films.

Table S3. Characteristics of perovskite solar cells based on Control, $\mathrm{CsCl}$ and $\mathrm{CsCl} / \mathrm{CH}_{3} \mathrm{O}-$ PEAI perovskite films, under reverse voltage scanning.

Table S4. The photovoltaic parameters for PSC devices based on Control, $\mathrm{CsCl}$ and $\mathrm{CsCl} / \mathrm{CH}_{3} \mathrm{O}-\mathrm{PEAI}$ perovskites films which extracted from measuring $J-V$ curves at simulated one sun illumination (AM 1.5G) performed by reverse and forward.

Table S5. The fitted parameters for IS measurements PSCs based on Control, $\mathrm{CsCl}$ and $\mathrm{CsCl} / \mathrm{CH}_{3} \mathrm{O}-\mathrm{PEAI}$ perovskite films.

Figure S1. Top-view SEM images of perovskite films (a) Control, (b) $\mathrm{CsCl}$, and (c) $\mathrm{CsCl} / \mathrm{CH}_{3} \mathrm{O}-\mathrm{PEAI}$

Figure S2. AFM 3D images of perovskite films (a) Control, (b) $\mathrm{CsCl}$, and (c) $\mathrm{CsCl} / \mathrm{CH}_{3} \mathrm{O}-$ PEAI.

Figure S3. Tauc plot results of the Control, $\mathrm{CsCl}$ and $\mathrm{CsCl} / \mathrm{CH}_{3} \mathrm{O}-\mathrm{PEAI}$ perovskites films $\mathrm{S}-6$

Figure S4. Steady-state photocurrent output of PSCs (a) Control, (b) $\mathrm{CsCl}$, and (c) $\mathrm{CsCl} / \mathrm{CH}_{3} \mathrm{O}-\mathrm{PEAI}$.

Figure S5. Photovoltaic parameters of a) $V_{o c}$, b) $J_{s c}$, c) FF, and d) PCE for PSCs based on Control, $\mathrm{CsCl}$ and $\mathrm{CsCl} / \mathrm{CH}_{3} \mathrm{O}-\mathrm{PEAI}$ perovskites films.

Figure S6. The enlarged Nyquist plots of PSCs based on Control, $\mathrm{CsCl}$ and $\mathrm{CsCl} / \mathrm{CH}_{3} \mathrm{O}-$

PEAI perovskites films. 
Table S1. The fitted parameters of TRPL spectra (excitation at $475 \mathrm{~nm}$ ) of Control, $\mathrm{CsCl}$ and $\mathrm{CsCl} / \mathrm{CH}_{3} \mathrm{O}-\mathrm{PEAI}$ perovskite films deposited on quartz glass.

\begin{tabular}{lccccc}
\hline \multicolumn{1}{c}{ Sample } & $\square \tau_{\text {ave }}(\mathrm{ns})$ & $\square \square \mathrm{A}_{1}$ & $\square \tau_{1}(\mathrm{~ns})$ & $\square \square \mathrm{A}_{2}$ & $\square_{\square} \tau_{2}(\mathrm{~ns})$ \\
\hline Control & 767.28 & 127.69 & 54.93 & 805.95 & 775.28 \\
$\mathrm{CsCl}$ & 1085.56 & 112.96 & 162.30 & 828.60 & 1104.06 \\
$\mathrm{CsCl} / \mathrm{CH}_{3} \mathrm{O}-\mathrm{PEAI}$ & 1248.26 & 103.34 & 637.41 & 808.71 & 1286.92 \\
\hline
\end{tabular}

Table S2. Calculated perameters and trap density (Nt) of the Control, $\mathrm{CsCl}$ and $\mathrm{CsCl} / \mathrm{CH}_{3} \mathrm{O}-$ PEAI perovskite films.

\begin{tabular}{lllll}
\hline & $L(\mathrm{~nm})$ & $\varepsilon$ & $V_{\mathrm{TFL}}(\mathrm{V})$ & $N \mathrm{t}\left(\times 10^{15} \mathrm{~cm}^{-3}\right)$ \\
\hline Control & 700 & 42.3 & 0.47 & 4.49 \\
$\mathrm{CsCl}$ & 700 & 42.3 & 0.34 & 3.25 \\
$\mathrm{CsCl} / \mathrm{CH}_{3} \mathrm{O}-\mathrm{PEAI}$ & 700 & 42.3 & 0.22 & 2.1 \\
\hline
\end{tabular}

Table S3. Characteristics of perovskite solar cells based on Control, $\mathrm{CsCl}$ and $\mathrm{CsCl} / \mathrm{CH}_{3} \mathrm{O}-$ PEAI perovskite films, under reverse voltage scanning.

\begin{tabular}{lcccc}
\hline & $J_{s c}\left(\mathrm{~mA} / \mathrm{cm}^{2}\right)$ & $V_{o c}(\mathrm{~V})$ & FF (\%) & PCE (\%) \\
\hline Control & $24.00 \pm 0.03$ & $1.109 \pm 0.008$ & $74.1 \pm 0.25$ & $19.72 \pm 0.4$ \\
$\mathrm{CsCl}$ & $24.43 \pm 0.02$ & $1.154 \pm 0.006$ & $78.9 \pm 0.23$ & $22.24 \pm 0.3$ \\
$\mathrm{CsCl} / \mathrm{CH}_{3} \mathrm{O}-\mathrm{PEAI}$ & $24.66 \pm 0.03$ & $1.186 \pm 0.005$ & $79.5 \pm 0.20$ & $23.25 \pm 0.3$ \\
\hline
\end{tabular}


Table S4. The photovoltaic parameters for PSC devices based on Control, $\mathrm{CsCl}$ and $\mathrm{CsCl} / \mathrm{CH}_{3} \mathrm{O}-\mathrm{PEAI}$ perovskites films which extracted from measuring $J-V$ curves at simulated one sun illumination (AM 1.5G) performed by reverse and forward.

\begin{tabular}{llllll}
\hline & Direction & $J_{\mathrm{SC}}\left(\mathrm{mA} / \mathrm{cm}^{2}\right)$ & $V_{\mathrm{OC}}(\mathrm{V})$ & FF (\%) & PCE (\%) \\
\hline Control & Reverse & 24.00 & 1.109 & 74.1 & 19.72 \\
& Forward & 23.55 & 1.084 & 68.0 & 17.36 \\
$\mathrm{CsCl}$ & Reverse & 24.43 & 1.154 & 78.9 & 22.24 \\
& Forward & 24.31 & 1.139 & 76.2 & 21.10 \\
$\mathrm{CsCl} / \mathrm{CH}_{3} \mathrm{O}-\mathrm{PEAI}$ & Reverse & 24.66 & 1.186 & 79.5 & 23.25 \\
& Forward & 24.47 & 1.172 & 78.2 & 22.43 \\
& & & & & \\
\hline
\end{tabular}

Table S5. The fitted parameters for IS measurements PSCs based on Control, CsCl and $\mathrm{CsCl} / \mathrm{CH}_{3} \mathrm{O}-\mathrm{PEAI}$ perovskite films.

\begin{tabular}{lll}
\hline & $R_{\mathrm{s}}\left(\Omega \mathrm{cm}^{2}\right)$ & $R_{\mathrm{rec}}\left(\Omega \mathrm{cm}^{2}\right)$ \\
\hline Control & 21.9 & 2394.6 \\
$\mathrm{CsCl}$ & 10.7 & 5133.3 \\
$\mathrm{CsCl} / \mathrm{CH}_{3} \mathrm{O}-\mathrm{PEAI}$ & 6.6 & 6479.7 \\
\hline
\end{tabular}



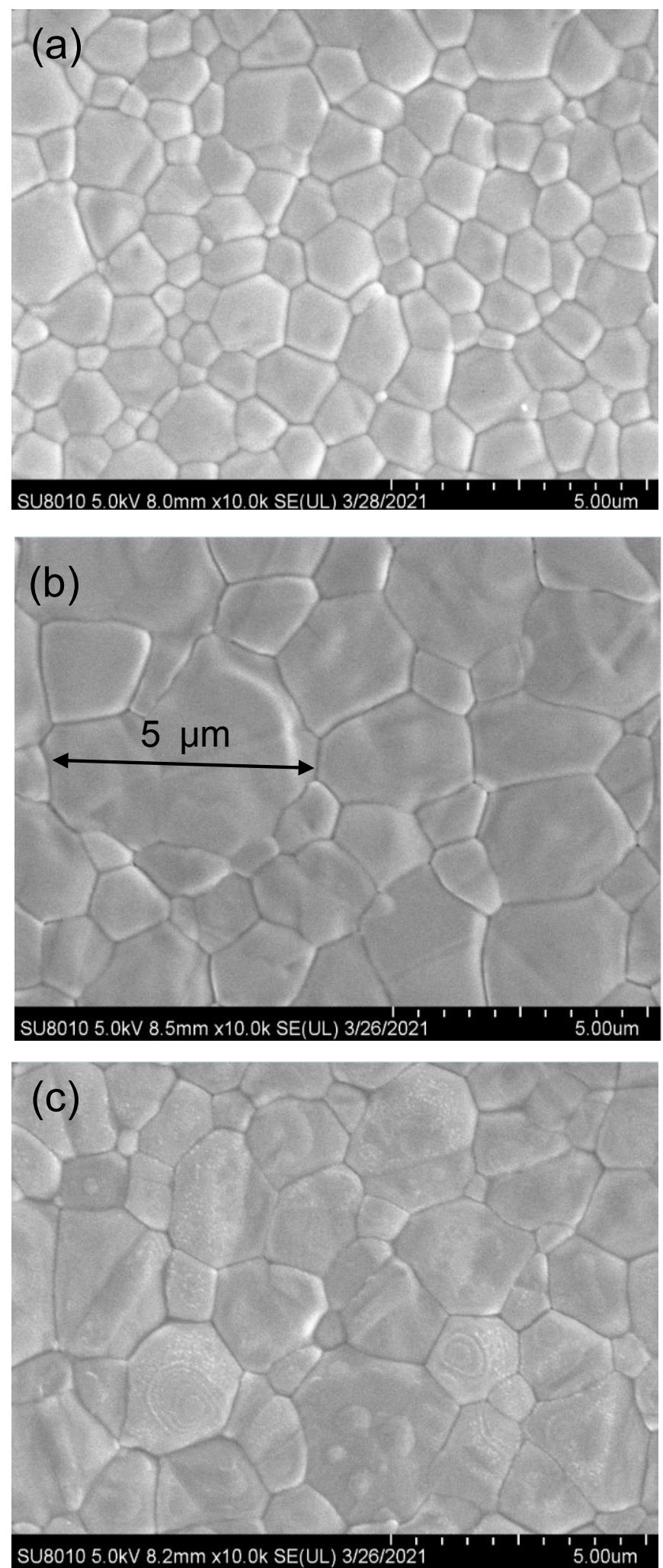

Figure S1. Top-view SEM images of perovskite films (a) Control, (b) $\mathrm{CsCl}$, and (c) $\mathrm{CsCl} / \mathrm{CH}_{3} \mathrm{O}$-PEAI 
(a)

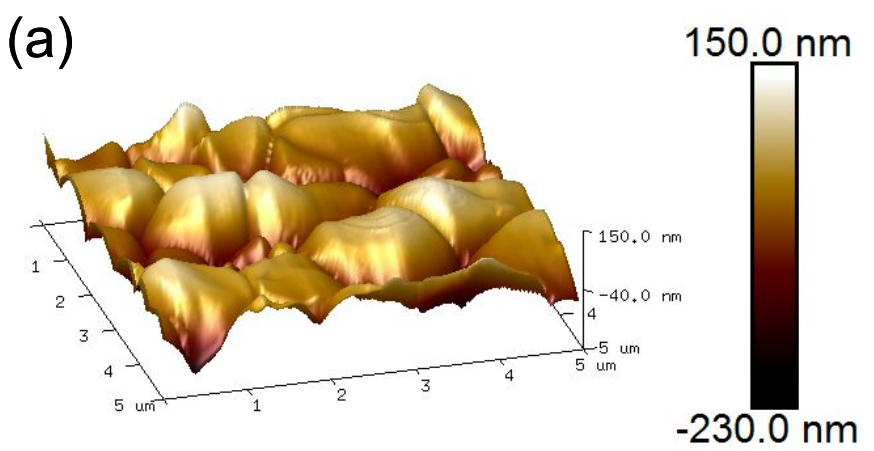

Height Sensor

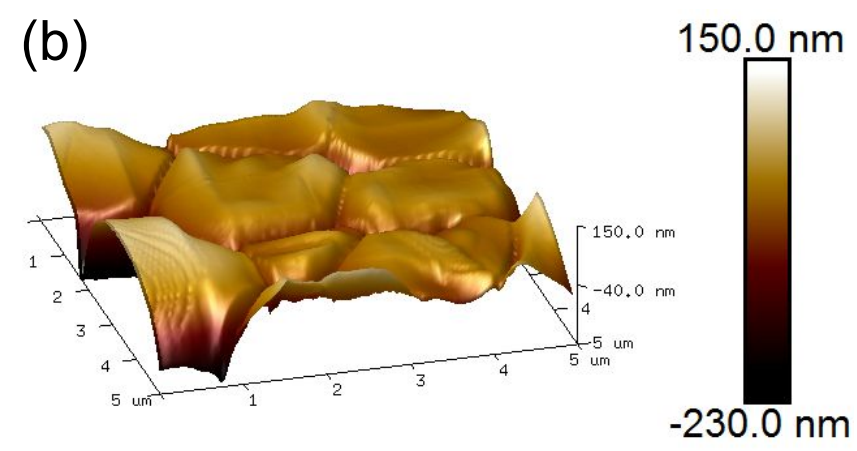

Height Sensor

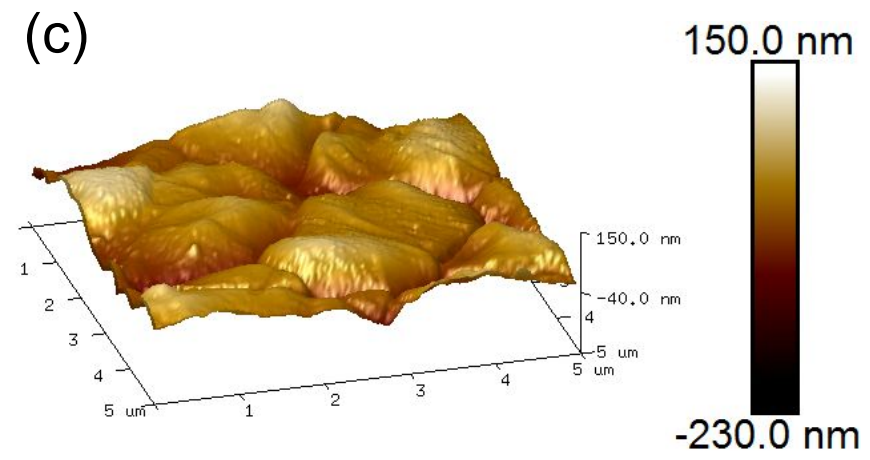

Height Sensor

Figure S2. AFM 3D images of perovskite films (a) Control, (b) $\mathrm{CsCl}$, and (c) $\mathrm{CsCl} / \mathrm{CH}_{3} \mathrm{O}-$ PEAI. 


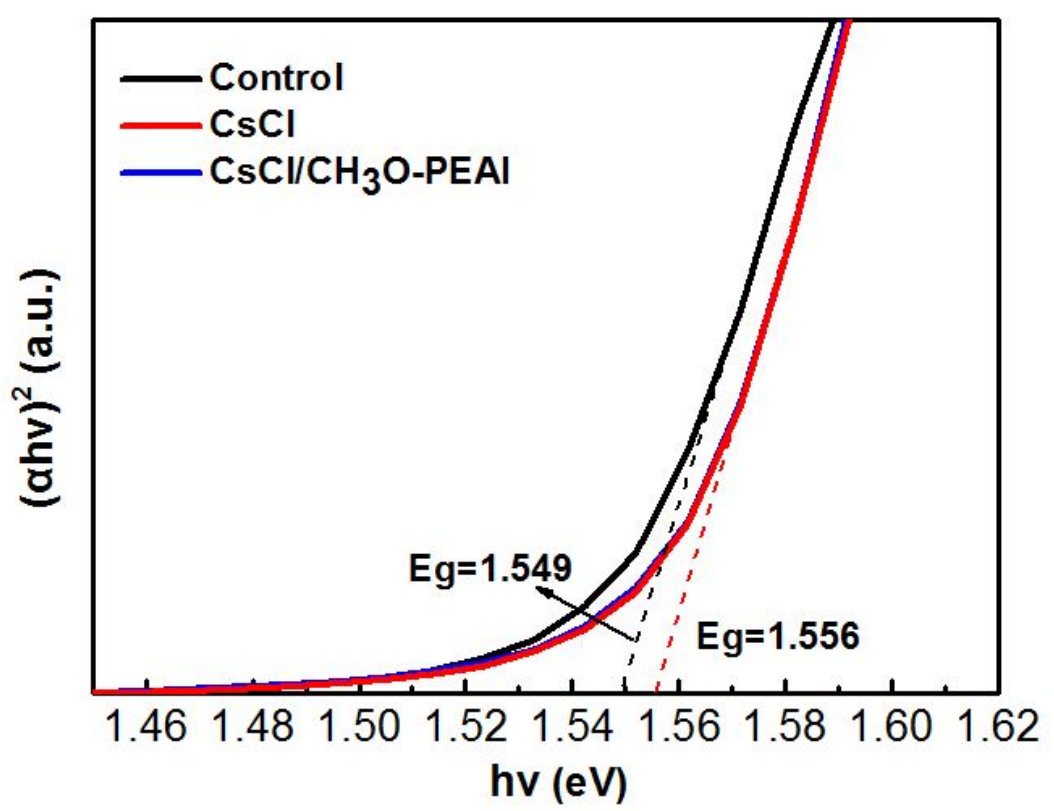

Figure S3. Tauc plot results of the Control, $\mathrm{CsCl}$ and $\mathrm{CsCl} / \mathrm{CH}_{3} \mathrm{O}-\mathrm{PEAI}$ perovskites films 
(a)

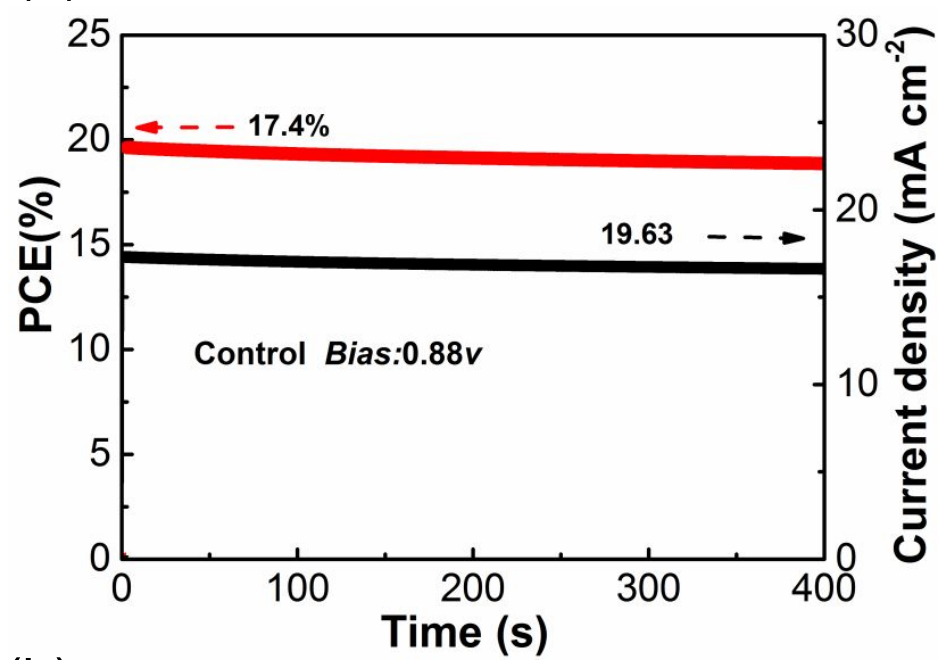

(b)

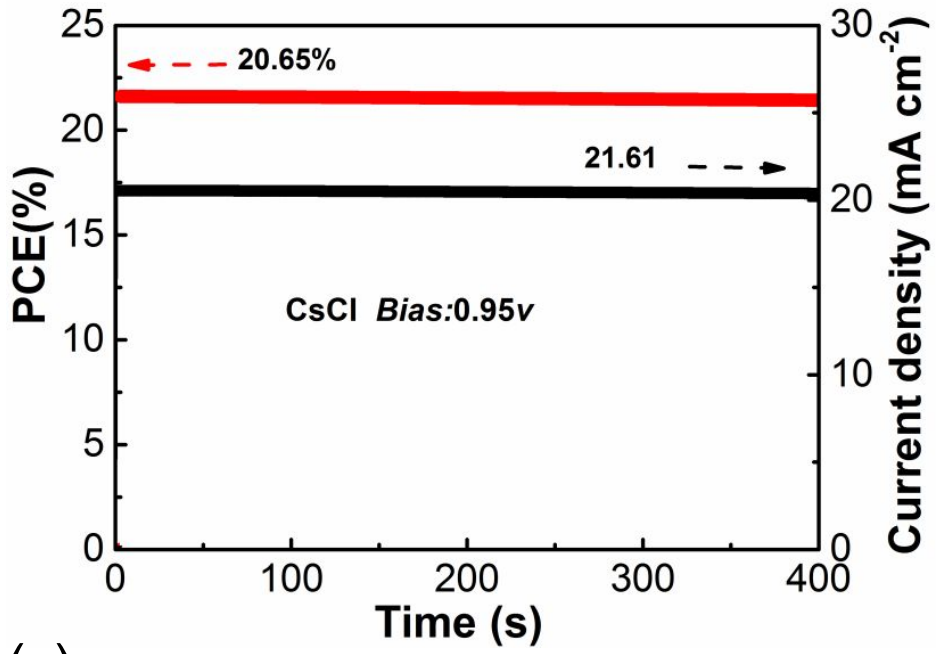

(c)

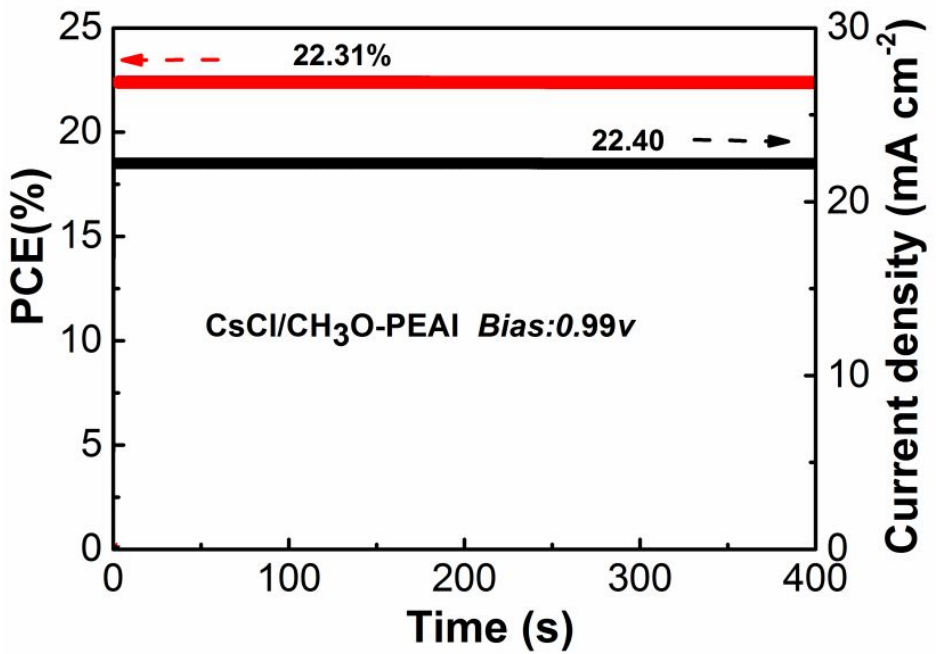

Figure S4. Steady-state photocurrent output of PSCs (a) Control, (b) $\mathrm{CsCl}$, and (c) $\mathrm{CsCl} / \mathrm{CH}_{3} \mathrm{O}-\mathrm{PEAI}$. 

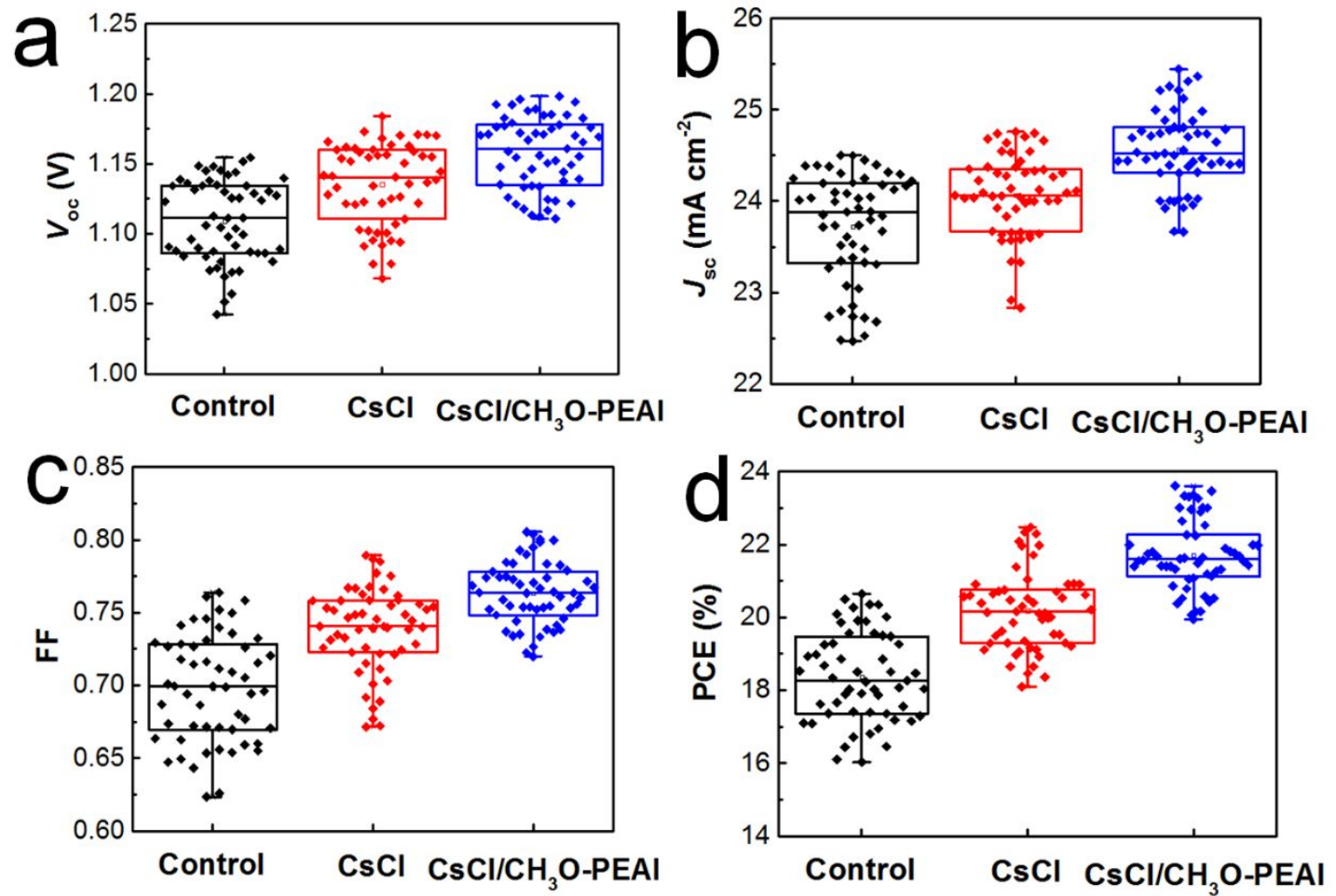

Figure S5. Photovoltaic parameters of (a) $V_{o c}$, (b) $J_{s c}$, (c) FF, and (d) PCE for PSCs based on Control, $\mathrm{CsCl}$ and $\mathrm{CsCl} / \mathrm{CH}_{3} \mathrm{O}-\mathrm{PEAI}$ perovskites films. 


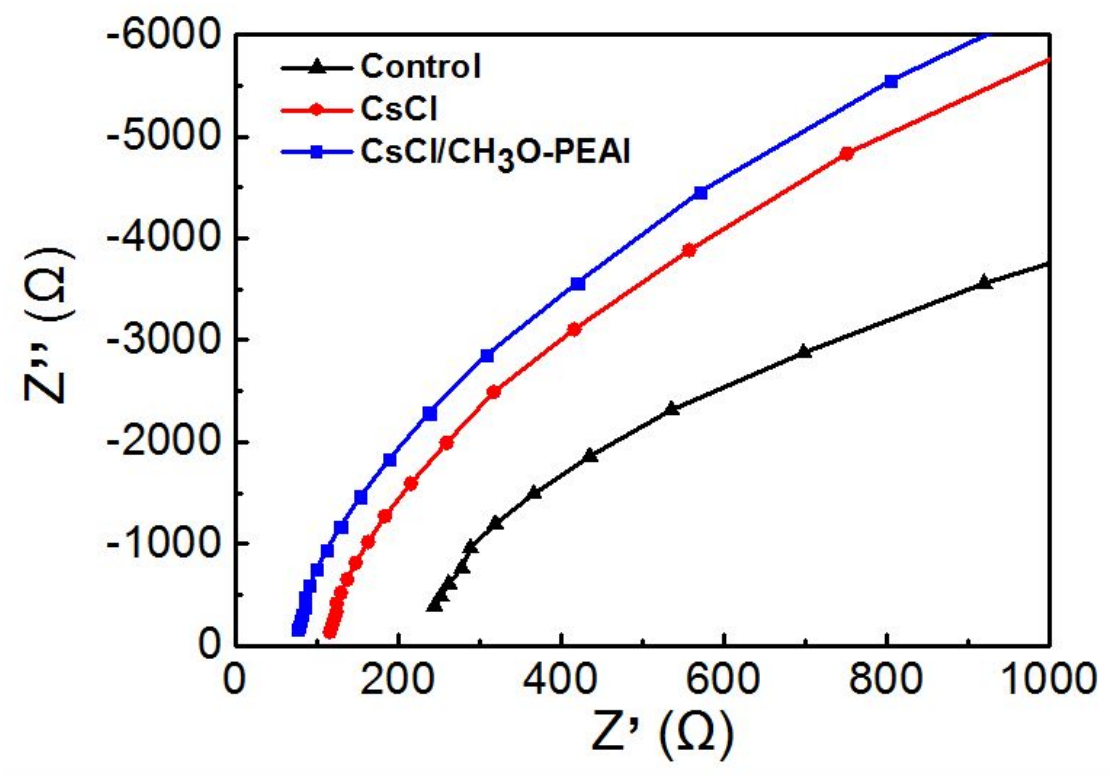

Figure S6. The enlarged Nyquist plots of PSCs based on Control, $\mathrm{CsCl}$ and $\mathrm{CsCl} / \mathrm{CH}_{3} \mathrm{O}-$ PEAI perovskites films. 